\title{
Rapid adaptation to a prismatic distortion
}

\author{
HANS WALLACH \\ Swarthmore College, Swarthmore, Pennsylvania 19081
}

and

\author{
EUGENIE WALSH FLAHERTY \\ Hahnemann Community Mental Health/Mental Retardation Center, Philadelphia, Pennsylvania 19102
}

\begin{abstract}
Among several shape distortions caused by a wedge prism is one where a pattern of evenly spaced lines that are parallel to the prism base reaches the eye with distances seen through the base region contracted and distances seen through the apex region expanded. Rapid partial adaptation to this distortion was obtained by having subjects who wore the prism in vertical orientation move the head up and down during the exposure period. The head movements caused the distortion to shift up and down through the line pattern. When, with subjects' heads stationary, the distortion was made to shift up and down by artificial means, rapid adaptation failed to develop. It is proposed that head movements are here needed for adaptation to develop, because the covariance between them and the shifting of the distortion serves as a signal that the distortions are not objective facts and causes the initiation of the adaptation process.
\end{abstract}

Wallach and Barton (1975) demonstrated rapid partial adaptation to spectacles that, by stereoscopic means, made a frontal plane appear to have a concave shape, like a piece of a large cylindrical surface with horizontal axis, seen from the inside. These spectacles essentially consisted of a wedge prism in front of each eye, with the prism bases in vertical orientation and toward the temples. The mild distortion that these prisms produce (vertical lines, e.g., become slightly curved) thus had different orientations in the two eyes and caused the described stereoscopic effect. Adaptation to these spectacles was easily demonstrated: if, after an adaptation period, subjects looked without the spectacles at a plane patterned surface, the surface appeared to have a convex curvature.

This adaptation was measured by giving a flexible patterned surface the concave curvature that compensated for the perceived convexity and looked plane to the subject. Initially, there were two exposure conditions: a subject, wearing the spectacles sat in front of a wall covered with an irregular pattern of dots, either with his head stationary in a headrest or continually nodding his head up and down. In the first condition, the spectacles caused the wall to appear as a rigid concave surface; the nodding in the second condition caused the concavity to move up and down and continuously deform the apparent shape of the wall. Adaptation was obtained only in the latter condition.

The question arose as to whether nodding in the exposure condition caused adaptation merely because of the deformation of the surface, the shifting up and down of the stereoscopic concavity, or whether the

This work was supported by Grant $25,000 \mathrm{X}$ from the National Science Foundation to Swarthmore College, Hans Wallach, principal investigator. nodding of the head played a role beyond being merely the optical cause for the deformation. Therefore, the wall was replaced by a plane board showing a similar dot pattern; it was made to move up and down while the subject observed it through the spectacles. Here, as in the second condition, the concavity shifted through the surface and deformed it, but the nodding head movement was absent. Very little, if any, adaptation was obtained after exposure to this condition.

What is the meaning of this peculiar result? The role that head movements played here was not merely that they caused the deformations of the surface; their presence proved essential for the development of rapid adaptation. We believe that they cause the adaptation process by giving rise to a signal that the deformations are not genuine environmental events. They do this by being covariant with these deformations. Sensory inputs representing environmental motions that are covariant with bodily movements are usually caused by these movements. Were such environmental motions perceived, they would have to be cognitively discounted. Having them eliminated at the level of perception by adaptation helps make perception veridical. An adaptation process that is instigated by covariance between these environmental motions and bodily movements serves this purpose. ${ }^{1}$

The hypothesis that covariance between a sensory input and bodily movements operates as a signal that instigates an adaptation process has a wider application. Every adult is equipped with a number of compensating processes that prevent certain visual inputs that result from his own movements from being perceived as motions in the visual environment. The constancy of visual direction is an example. When one turns one's head to the right and thereby causes the stationary visual environment to become displaced to 
the left relative to the head, stimulation is produced that under other circumstances would result in a perceived displacement of the environment to the left. An existing compensation process prevents one from actually perceiving this displacement. This compensation is a very accurate function, as measurements by Wallach and Kravitz $(1965,1968)$ have shown. There is also an existing compensation that prevents one from perceiving the rotation relative to the eye of objects past which one walks (Wallach, Stanton, Becker. 1974). Recently. Wallach and Flaherty (1975) demonstrated an existing compensation for the expanding motion associated with the growth of retinal images of objects toward which one moves.

There is little doubt that these compensating processes were originally learned. We believe that this learning took place because the covariance between the visual inputs and the bodily movements that caused them served as a signal to the nervous system to develop a compensation for these particular visual inputs. Unfortunately, this role of covariance is not as obvious here as it is in the experiment by Wallach and Barton. This will become clear when we discuss another kind of experimental adaptation where the covariance principle is applicable. This adaptation process is the experimental modification of the constancy of visual direction (CVD). The latter, as we have seen, prevents field displacements, relative to the head and caused by head movements, from being perceived. In experimental adaptation in CVD. the subject learns to compensate for actually given displacements of the visual field. Devices are employed that cause, by various means, field displacements during and dependent on head movements. With prolonged exposure, there artificial field displacements gradually become perceived to a diminished degree and, when adaptation is complete, the subject is not aware of them at all. Reversing prisms. magnifiers or minifiers, have been employed to cause displacements of the visual field during head movements, and mechanical devices have also been used. ${ }^{2}$ All of these devices cause a shifting of the visual field only when the head is turned. The field displacements for which compensation develops are therefore strictly covariant with the head movements.

Here, however, it is impossible to know whether covariance plays a specific role in the adaptation process, for the head movements that are covariant with the field displacements must be present because they cause the field displacements. In the Barton experiment, on the other hand, the input for which partial adaptation develops exists independently of head movements. The plane pattern on which the subject observes the effect of the spectacles can be made to deform by being moved up and down. But rapid adaptation develops only when nodding movements are made. The head movements are shown to be a necessary condition for the development of compensation. If they have this effect by virtue of their covariance with the pattern deformation, the causal role of this covariance is clear. Covariance may play the same role in adaptation to field displacements caused by head movements. But such a causal role of the covariance is here not obvious. since the head movements are necessary to cause the stimulation to which the subject adapts.

The same considerations apply to the original acquisition of CVD, which probably came about in the same fashion as experimental adaptation in CVD, and to the two other existing compensation processes mentioned. Here, too, it can be assumed that covariance with movements of the subject instigated the development of a compensation process and selected the sensory input to be dealt with. But the causal role of movements cannot be established, because without the subject's movements there is no sensory input to be compensated for. Only in the Barton experiment is there evidence for a causal role of head movements.

Because Barton's experiment is such a decisive factor in the formulation of the covariance principle, we have searched for other kinds of adaptation where the covariance principle plays an analogous role. The experiment to be reported resulted from this search.

When a wedge prism is located close to the eye and centered on the pupil, the displacement of the lines of sight by the prism varies with the angle at which they cross it. Those that pass near the apex are more displaced than those that pass perpendicularly, and those that pass near the base are displaced less. This causes a shape distortion such that, in a pattern of regularly spaced lines that are parallel to the prism base, distances seen through the apex region are increased and distances seen through the base region are decreased.

Adaptation to this shape distortion has been found to be very slow. Pick and Hay $(1964,1966)$ obtained an adaptation amounting to 2.3 prism diopters after an exposure period lasting 21 days, but an exposure period of 3 days did not yield an adaptation. A search for specific adaptation conditions and their concentrated use in the exposure period seemed indicated. We felt that a condition where head movements cause continuous shifting of the distortion through a stationary pattern may hold the answer. Our first experiment was an attempt to obtain rapid adaptation when the shifting of the distortion varied with incessant head movements.

In preliminary experiments, a subject sat in front of a screen covered with evenly spaced horizontal stripes, wearing a long 30 diopter prism in vertical orientation, i.e., with base horizontal, in front of his right eye, with the left eye occluded. Viewed through the prism, the pattern of stripes looked distorted. When the prism was oriented with the base downward, the stripes and the intervals between them were narrower in the lower parts of the pattern, and the upper part of the pattern seemed expanded. When 
the subject nodded his head. this distortion appeared to travel up and down with the head movements. When, after an exposure period of $10 \mathrm{~min}$, in which the subjeet nodded his head continuously and observed the distortion travel up and down. the prism was taken off. the subject saw a mild distortion of the pattern. with the upper part now somewhat contracted and the lower part expanded. This observation indicated that partial adaptation to the distortion caused by the prism had taken place.

In the main experiments. this postexposure distortion was measured by compensation: a prism was selected from a graduated series that would cause the subject to perceive the stripe pattern as regular. In all experiments, during the test and exposure periods, the subject sat $165 \mathrm{~cm}$ from a large white screen. $154 \mathrm{~cm}$ high and $121 \mathrm{~cm}$ wide. The screen was covered with horizontal black stripes, $1.9 \mathrm{~cm}$ wide and spaced $3.1 \mathrm{~cm}$. In the center of the screen and at the subject's eye level was an aperture, $21 \times 12 \mathrm{~cm}$, in which the picture of a small TV set was visible.

During the adaptation period, the subject wore a uelder's headset to which a vertical plate was attached so that it covered part of his face in front of his eyes. The 30 diopter prism fitted into an oblong opening in that plate so that it was approximately centered on the subject's right eye. The Lucite prism, which was $7 \mathrm{~cm}$ long and $3 \mathrm{~cm}$ wide. could be inserted into the plate either with base upward or base downward. In each of our experiments. approximately half of our subjects adapted to a prism with base upward and the other half to a prism with base downward. A second headset with a similar vertical plate was used for the test. Six test prisms. ranging in strength from 2 to 12 diopters in steps of 2 diopters, were mounted side by side across a large opening in a second plate. It fitted into a recess in the plate that was attached to the headset and could be slid sideways so that each of the test prisms could be made to cover the aperture in front of the subject's eye. In both headsets. the aperture for the prism was covered with Wratten filter No. 58 to eliminate color fringes and increase acuity. The subject's left eye was covered throughout the experiment.

\section{EXPERIMENT I}

\section{Method}

An abbreviated method of limits was used in the pre- and postexposure tests. Before each trial, the subject shut his eyes while the experimenter shifted the appropriate test prism into position in front of the aperture. Then the subject had to fixate the TV set, nod his head up and down twice and observe whether or not there were width change in the stripe pattern. He closed his eyes again and reported his observations. The experimenter shifted the next prism into position, and the next trial began. The TV set was turned off during all testing.

In each test, the strongest prism was presented in the first trial. followed by the other prisms in descending diopter value. This was continued until the subject reported "no change in width" for two consecutive prisms. Then the ascending series was begun by presenting the third prism below the first prism that had elicited a "no-change" report in the descending series of trials. The ascending series was continued until width changes were reported tor two consecutive prisms. The average of the diopter values of the strongest prisms eliciting "no-change" reports in the descending and in the ascending series became a subject's test score: This procedure. then, yielded a measure of the dicpter value of the limit between "no-change" reports and those width changes that were produced ty the prism to which a subject adapted. The opposite width changes were hardly ever experienced during the tests. This means, in practice. that when a subject was scheduled to adapt to a prism with base upward. all the test prisms were also presented with base upward, and so forth. This procedure was dictated by our fear that giving a subject after the exposure period a test trial that would cause hin to perceive a width change opposite to the one to which he had adapted might diminish the adaptation effect on subsequent trials. This was avoided by starting all tests with a descending series and by determining only one of the limits of the "no-change" region. the one first encountered in the descending series. This procedure was permissible because we aimed to obtain adaptation scores: for this purpose. the difference between analogous pre- and postadaptation scores suffices.

After the preexposure test, the headset with the 30 diopter prism was put on the subject and he was asked to keep looking at the TV set and to move his head up and down. There was a brief rest after 5 min. after which the subject resumed his head nodding for another 5-min period. While the subject's eyes were closed, the experimenter exchanged headsets and the postexposure test was administered (Test A). When that test was completed and the headsets were exchanged, a second exposure period began, which lasted $20 \mathrm{~min}$, with a brief rest every $5 \mathrm{~min}$. After another test (Test B), the subject was asked to keep his eyes closed and sit still for $5 \mathrm{~min}$. A last test (Test C) was administered after this dissipation period. In this experiment, the room was well illuminated with daylight. Twenty-one paid undergraduates served as subjects. No subjects who wore eyeglasses participated.

\section{Results}

Three adaptation scores were computed for each subject. by subtracting from each one of his postexposure scores his preexposure score. The means of each of these three difference scores were computed. The three means and the level of their significance are presented in Table 1 under the headings of the postexposure tests to which they pertain. Signiticant adaptation effects were obtained after exposure periods of $10 \mathrm{~min}$ and of an additional 20 min amounting to about 2.5 prism diopters. The adaptation effect measured after $10 \mathrm{~min}$ amounted to $9 \%$ of full adaptation. The 5-min dissipation period that separated Test $C$ from Test $B$ caused a decrease in the adaptation effect of 1.43 diopters significant at the .01 level $[t(20)=2.66]$. The adaptation effect that remained was still significant $(t=1.8)$.

\section{Table 1}

Mean Shape Adaptation Effects After Exposure to Wedge Prism Measured in Prism Diopters $(\mathbf{N}=21)$

\begin{tabular}{lccc}
\hline & Test A & Test B & Test C \\
Exposure & After & After Additional & After \\
Condition & 10-Min Exposure & 20-Min Exposure & 5-Min Rest \\
\hline
\end{tabular}

\begin{tabular}{llll}
\hline Head Moving & & & \\
Mean & 2.76 & 2.48 & 1.05 \\
$\mathrm{t}$ & $3.57 * *$ & $3.08 * *$ & $1.81^{*}$ \\
\hline & ${ }^{*} p<.05$ & ${ }^{*} p<.005$ &
\end{tabular}




\section{EXPERIMENT II}

Our second experiment was designed to answer the question of whether head movements are necessary for this rapid development of adaptation to shape distortion, or whether the pattern deformations that these head movements produce are solely responsible. The same question had been raised in the experiment by Wallach and Barton. In their last experiment, they artificially produced the deformations that had previously occurred when head movements made the shape distortion caused by the spectacles shift up and down; rapid adaptation failed to develop. We did an analogous experiment in which we produced artificially the pattern deformations that take place when a subject viewed the striped field through the adaptation prism and moved his head up and down. These deformations, that is, the shifting of the pattern distortion up and down caused by the nodding, occur because these head movements cause a tilting of the prism in relation to the subject's line of sight. Hence, we provided an arrangement that tilted a prism located in front of the subject's stationary eye mechanically. The angle through which the prism was tilted and the rate of its motion duplicated the tilt angle and the tilt rate of the prism worn during head nodding. This arrangement presented the subject with the same kind of deformation that was previously produced by head nodding, but covariant head movements were absent as they were in the last experiment by Wallach and Barton, and we did not expect this exposure condition to yield rapid adaptation. We shall refer to this arrangement where the prism tilts but the subject does not move his head as the stationary exposure condition.

It seemed to us that the tests connected with the stationary exposure condition should be modified to fit this condition. In every test trial in Experiment I, the subject fixated the TV set and nodded his head twice. Since there were no head movements in the stationary exposure condition, head movements had to be eliminated from the tests also. Therefore, the subject's head was kept immobile by a bite board during the tests, and he was instructed to run his eyes up and down the stripe pattern and to look for a difference in width between the upper and lower parts of the pattern. Because we felt that the same test procedure should be used in connection with the head-movement exposure condition, Experiment I was repeated and the new test conditions were employed. Illumination conditions were also changed to conform to those of the stationary exposure condition.

An additional feature was incorporated into the stationary condition. According to our interpretation, the presence of head movements is necessary for the development of rapid adaptation because the resultant proprioceptive stimulation is covariant with the sensory input for which compensation develops. The question may be raised whether any covariant stimulus input may have that effect. The nervous system may regard any pair of covariant stimuli as redundant information and start compensation for one of them. To check on this possibility, we arranged for the illumination of the stripe pattern to vary exactly with the up-and-down shifting of the pattern distortion. This was achieved by connecting to the mechanism that tilted the prism up and down a variable transformer that delivered the power for a projector that illuminated the striped field.

\section{Method}

Equipment. In the stationary condition, the subject's head was held in position by a bite board and was surrounded by a housing that kept his visual field dark except for what he saw through an oblong aperture directly in front of his right eye. The aperture was covered by a Wratten filter No. 58 . Immediately beyond it was the mounting for the tilting prism, into which the prism could be inserted either base up or base down.

A simple mechanism controlled both the tilt of the prism and the setting of the variable transformer. A linkage was eccentrically attached to the slow shaft of a dc shunt motor run on a Minarik speed control. This linkage was connected to an arm mounted on the shaft of the transformer and pushed and pulled it back and forth, thereby turning the transformer shaft. The linkage was also connected to an arm mounted on the shaft on which the prism turned, causing a synchronous turning of the prism. With each rotation of the motor, the prism tilted back and forth through an angle of $\mathbf{4 0} \mathrm{deg}$, which was the average angle of prism tilt when the subject nodded his head. Simultaneously, the transformer shaft turned back and forth between fixed points. These points were so chosen that the illumination delivered to the striped field by the projection lantern changed between .05 and $.22 \mathrm{fc}^{3}$

A 30 diopter prism displaces the visual field on the average by $16 \mathrm{deg}$. To cope with this fact, the tilting device was adjusted in such a way that the center of the striped field was optically on eye level when the prism was mounted base up. We compensated for the upward displacement of the field that took effect when the prism was mounted base down by putting into the light path between the prism and the striped field two first-surface mirrors. They were so tilted that the center of the field again appeared on eye level; they were removed when the prism was used base up.

Procedure. As stated, the tests employed in connection with the stationary and with the head-movement exposure conditions were identical. They differed from the tests employed in Experiment $I$ in three ways. The subject's head was held stationary by a bite board, and in each trial he looked for differences in the apparent width of the stripes and their intervals by scanning the field for $10 \mathrm{sec}$. In place of daylight illumination, the striped field was lit by the projection lantern, which delivered constant illumination of $.165 \mathrm{fc}$. While in Experiment $I$ all tests started with a series of trials in which the test prisms were employed in an order of descending diopter value, followed by the ascending series, 8 out of 16 subjects were first given an ascending series in all tests, that is, the test procedure was reversed. When our results showed that this procedure produced smaller adaptation effects, we added another 8 subjects, who served in the head-movement condition and started all tests with the descending series. This gave us a total of 16 subjects who were always tested with the descending series first. Their results could be compared with the results of Experiment I.

The exposure period for the head-movement conditions was the same as in Experiment 1 , except that the same artificial illumination was used as in the tests. In the stationary exposure condition, the illumination varied with the prism's reciprocating tilting movements, one cycle lasting $2.7 \mathrm{sec}$, the same as the average rate of head nodding. Each subject served in both exposure 
conditions. At least $\mathbf{S}$ days intervened between the two experimental sessions. Eight subjects served first in the stationary condition and then in the head-movement condition; for the other eight subjects. this order was reversed. For four subjects in each order. the prism was base up in both sessions, and for the other four subjects, it was base down. In all other respects, the procedures were analogous to that of Experiment I.

\section{Results}

As was done in presenting the results for Experiment I, mean adaptation scores are presented in Table 2 under the headings of the postexposure tests from which they were derived. With the original group of 16 subjects, the 20-min exposure to the head-movement condition produced a significant mean adaptation effect as measured by the immediate test. After the 5-min dissipation period, this mean effect remained unaltered and significant. No adaptation effects were obtained with the stationary exposure condition. The small increase after the 5-min dissipation period was not significant. The mean adaptation effect produced by the headmovement condition, as measured after the 20 -min exposure period, was significantly different from the corresponding result after the stationary exposure condition $[\mathrm{t}(15)=2.7, \mathrm{p}<.01]$. This outcome is analogous to that obtained by Wallach and Barton with spectacles that caused stereoscopic concavity.

There was a conspicuous difference between the adaptation effects produced by the head-movement conditions in Experiment $I$ and Experiment II after the $10-\mathrm{min}$ exposure period. Comparison with the results obtained from the 16 subjects for whom tests started with the descending series shows that this was due to the test procedure in which half the subjects were started with the ascending series. The mean adaptation effect of 1.97 diopters obtained from these subjects is not conspicuously different from the corresponding mean of 2.76 diopters obtained in Experiment I.

The results obtained for the head-movement condition of Experiment II differed from the results of Experiment I in a peculiar way. In Experiment I, the 5-min dissipation period had brought a significant decrease of the mean adaptation score amounting to 1.43 diopters. No such dissipation effect was found in Experiment II, and this difference was significant: We compared the difference between the mean scores obtained with Tests B and C of Experiment I, where all subjects were tested starting with the descending series, with the corresponding difference obtained for the 16 subjects who in Experiment II adapted with head moving and for whom tests also started with the descending series. We found the two differences to be significantly different at the .03 level in a two-tailed test. Where these two groups of subjects are concerned, Experiments I and II differed only in two ways. One was the lower illumination in Experiment II, and the other was a difference in the
Table 2

Mean Shape Adaptation Effects After Exposure to Wedge Prism Measured in Prism Diopters (N = 16)

\begin{tabular}{|c|c|c|c|}
\hline $\begin{array}{l}\text { Exposure } \\
\text { Condition }\end{array}$ & $\begin{array}{c}\text { Test A } \\
\text { After } \\
\text { 10-Min Exposure }\end{array}$ & $\begin{array}{c}\text { Test B } \\
\text { After Additional } \\
\text { 20-Min Exposure }\end{array}$ & $\begin{array}{c}\text { Test C } \\
\text { After } \\
\text { 5-Min Rest }\end{array}$ \\
\hline \multicolumn{4}{|c|}{ Head Moving } \\
\hline Mean & .88 & 1.91 & 1.91 \\
\hline$t$ & .82 & $2.21 *$ & $2.81 * *$ \\
\hline \multicolumn{4}{|c|}{ Head Stationary } \\
\hline $\begin{array}{l}\text { Mean } \\
t\end{array}$ & .13 & 0 & $\begin{array}{l}.92 \\
.92\end{array}$ \\
\hline
\end{tabular}

Subjects Start With Descending Series

\begin{tabular}{lcll}
$\begin{array}{lll}\text { Head Moving } \\
\text { Mean }\end{array}$ & 1.97 & 1.79 & 1.84 \\
$\mathrm{t}$ & $2.2^{*}$ & $2.2^{*}$ & $2.26^{*}$ \\
\hline & $* p<.025$ & $* * 0.01$ &
\end{tabular}

test procedure. In Experiment I, subjects moved their heads up and down during the tests, whereas in Experiment II they kept their heads still. The fact that we obtained spontaneous dissipation for the effect tested with head movements and no dissipation with the other test may well indicate that these tests measure two different manifestations of adaptation.

\section{DISCUSSION}

Our experiment turned up a second case of adaptation to spectacles where rapid compensation develops only when head movements were present. In both instances, head movements caused the shape distortions produced by the spectacles to manifest themselves as pattern deformations. But that was not their only function. Producing these deformations by artificial means did not succeed in bringing about rapid adaptation. Head movements had to accompany the deformation. We propose that the covariance between the deformations and the head movements serves as a signal that the deformations are not genuine environmental facts and instigate the development of a compensation process that diminishes and perhaps ultimately eliminates the effects of these sensory inputs from perception."

We also propose that this effect of covariance is responsible for the original development of a number of other compensation processes with which everyone is equipped. They eliminate the effect of stimulation by visual motions that are caused by one's own movements.

Because it selects those sensory inputs that are caused by the observer's own movements and eventually results in freeing perceptual experience of immaterial content, initiation of compensation by covariance is a useful principle. Although it is easy to see that covariance is a good indicator for those sensory inputs that result from one's own movements, such a role of covariance is not obvious enough to 
favor the assumption that it becomes established by some learning process. Rather, we believe that this role is built into the nervous system. By being responsible, for the original development of CVD, of the compensation for movement-produced changes in object orientation, of the compensation for field expansion caused by moving forward, and perhaps of others, the initiation of compensation by covariance, in conjunction with a more general capacity for perceptual learning, does a job that otherwise would require the existence of a number of different innate compensation processes.

\section{REFERENCES}

Prck, H. L., JR., \& HuY, J. C. Adaptation to prismatic distortion. Psychonomic Science, 1964, 1, 199-200.

Pick, H. L.. JR., \& HAY, J. C. Gaze contingent adaptation to prismatic spectacles. American Journal of Psychology, $1966,79,443-450$.

Posin, R. L. Perceptual adaptation to contingent visual-field movement: An experimental investigation of position constancy. Doctoral dissertation, Yeshiva University, 1966.

Rock, I. The nature of perceptual adaptation. New York: BASIC Books, 1966.

Stratton, G. M. Vision without inversion of the retinal image. Psychological Review. 1897, 4. 341-360, 463-481.

von Holst, E., \& Mittelstaedt, H. Das Reafferenz-prinzip. Die Naturwissenschaften, 1950, 20, 464-467.

Wallach, H., \& Barton, W. Adaptation to optically produced curvature of frontal planes. Perception \& Psychophysics, 1975 , 18. $21-25$.

Wallach, H., \& Flaherty, E. W. A compensation for field expansion caused by moving forward. Perception \& Psychophysics, 1975, 17, 445-449.

Wallach, H., \& Kravitz, J. H. The measurement of the constancy of visual direction and of its adaptation. Psychonomic Science, 1965, 2, 217-218.
Wallach, H., \& Kravitz, J. H. Adaptation in the constancy of visual direction tested by measuring the constancy of auditory direction. Perception \& Psychophysics. 1968, 4, 299-303.

Wallach. H.. Stanton, L., \& Becker. D. The compensation for movement-produced changes in object orientation. Perception \& Psychophysics, 1974, 15, 339-343.

Wallach, H., Yablick, G. S.. \& Smith, A. Target distance and adaptation in distance perception in the constancy of visual direction. Perception \& Psychophysics, 1972. 12. 139-145.

\section{NOTES}

1. The covariance principle here described differs from the reafference principle of von Holst and Mittelstaedt (1950) and from Rock's concomitance principle (Rock. 1966, p. 85). The latter are concerned with an existing compensation for movement-produced stimulation and present a hypothesis about the nature of that compensating process. The covariance principle is concerned with its cause. Covariance selects the sensory input for which compensation eventually develops and initiates this development.

2. Adaptation to field displacement during head movements was discovered by Stratton (1897) when he wore inverting lenses. Partial adaptation was first demonstrated by Wallach and Kravitz (1965) and by Posin (1966). Wallach and Kravitz (1968) also developed a method for measuring partial adaptation and employed it for an investigation into the nature of this adaptation. For a listing of further work on this kind of adaptation, see Wallach, Yablick, and Smith (1972).

3. We are grateful to Otto Hebel for constructing this apparatus.

4. Whether only head movements can function in this fashion or whether other covariant movements would serve also must remain an open question.

(Received for publication September 10, 1975; revision received December 16. 1975.) 